\title{
Influence of hepatic steatosis on the outcomes of patients with chronic hepatitis B treated with entecavir and tenofovir
}

\author{
David Sooik Kim ${ }^{1, *}$, Mi Young Jeon ${ }^{1,2,3, *}$, Hye Won Lee, ${ }^{1,2,3}$, Beom Kyung Kim ${ }^{1,2,3}$, Jun Yong Park ${ }^{1,2,3}$, Do Young Kim ${ }^{1,2,3}$, \\ Sang Hoon Ahn $n^{1,2,3}$, Kwang-Hyub Han ${ }^{1,2,3}$, and Seung Up Kim ${ }^{1,2,3}$ \\ 'Department of Internal Medicine and ${ }^{2}$ Institute of Gastroenterology, Yonsei University College of Medicine, Seoul; ${ }^{3}$ Yonsei Liver Center, \\ Severance Hospital, Seoul, Korea
}

Background/Aims: The influence of hepatic steatosis (HS) on chronic hepatitis B (CHB) is unclear. We evaluated the influence of the degree of $\mathrm{HS}$, assessed using the controlled attenuation parameter (CAP) of transient elastography (TE), on treatment outcomes in $\mathrm{CHB}$ patients initiated on antiviral therapy.

Methods: A total of 334 patients who were initiated on entecavir or tenofovir between 2007 and 2016 with available TE results were recruited.

Results: Of the total study population, 146 (43.7\%) patients had HS (CAP $>238 \mathrm{~dB} / \mathrm{m})$. Three-hundred-three patients (90.7\%) achieved complete virological response (CVR) (hepatitis B virus DNA $<12$ IU/L), and 25 patients (7.5\%) developed hepatocellular carcinoma (HCC). Among hepatitis B e antigen (HBeAg)-positive patients ( $n=172,51.5 \%), 37$ (21.5\%) experienced $\mathrm{HBeAg}$ loss. On univariate analysis, CAP value was not associated with the probability of HCC development $(P=0.380)$. However, lower CAP value was independently associated with higher probability of HBeAg loss among HBeAg-positive patients (hazard ratio $[\mathrm{HR}]=0.991, P=0.026$ ) and with $\mathrm{CVR}$ achievement in the entire study population ( $\mathrm{HR}=0.996, P=0.004)$. The cumulative incidence of $\mathrm{HBeAg}$ loss among HBeAg-positive patients was significantly higher in patients without HS than in those with HS (log-rank, $P=0.022$ ).

Conclusions: CAP values were not correlated with HCC development in patients initiated on entecavir and tenofovir. However, CAP values were negatively correlated with the probability of $\mathrm{HBeAg}$ loss among $\mathrm{HBeAg}$-positive patients and with CVR achievement. (Clin Mol Hepatol 2019;25:283-293)

Keywords: Fatty liver; Hepatitis B, Chronic; Antiviral agent

\footnotetext{
Study Highlights

- There is a lack of evidence to support the influence of hepatic steatosis (HS) on antiviral therapy with entecavir and tenofovir.

- The controlled attenuation parameter (CAP) values, measured by transient elastography, can assess the degree of HS.

- CAP values were negatively correlated with the probability of hepatitis B e antigen (HBeAg) loss among HBeAg-positive patients.

- CAP values were associated with the achievement of complete virological response, whereas they were not associated with the risk of developing hepatocellular carcinoma.
}

\footnotetext{
Abbreviations:

ALT, alanine aminotransferase; AVT, antiviral therapy; BMI, body mass index; CAP, controlled attenuation parameter; $\mathrm{CHB}$, chronic hepatitis $\mathrm{B} ; \mathrm{Cl}$, confidence interval; $C V R$, complete virological response; $\mathrm{dB} / \mathrm{m}$, decibels per meter; $\mathrm{HBeAg}$, hepatitis B e antigen; HBV, hepatitis B virus; HCC, hepatocellular carcinoma; HS, hepatic steatosis; HR, hazard ratio; IQR, interquartile range; $\mathrm{KPa}$, kilopascals; LS, liver stiffness; TE, transient elastography

* David Sooik Kim and Mi Young Jeon contributed equally to this work.

Corresponding author : Seung Up Kim

Department of Internal Medicine, Yonsei University College of Medicine, 50-1 Yonsei-ro, Seodaemun-gu, Seoul 03722, Korea

Tel: +82-2-2228-1982, Fax: +82-2-393-6884

E-mail: ksukorea@yuhs.ac

https://orcid.org/0000-0002-9658-8050 


\section{INTRODUCTION}

More than 250 million people are infected with hepatitis B virus (HBV) worldwide, including $>6 \%$ of the Asian and Western Pacific adult population. ${ }^{1}$ Additionally, more than $20 \%$ of patients with chronic hepatitis B (CHB) developed hepatocellular carcinoma (HCC) within 10 years. ${ }^{2,3}$ Thus, to prevent poor outcomes in patients with HBV viremia due to uncontrolled replication, the primary treatment strategy is to suppress replication with antiviral therapy (AVT). ${ }^{4}$ Furthermore, newer AVT agents such as entecavir and tenofovir, with little to no resistance, introduce new methods of blocking HBV replication. ${ }^{5}$

Hepatic steatosis (HS) is frequently found in patients with chronic liver disease and its prevalence in HBV infected patients was reported to be between $14 \%$ and $67 \% .{ }^{6}$ However, the influence of HS on HBV infection has been controversial. First, no association between HS and HBV has been reported. Some studies showed no significant correlation between the presence of hepatitis $\mathrm{B}$ e antigen ( $\mathrm{HBeAg}$ ) and $\mathrm{HS}^{7,8}$ and between HBV DNA level and $\mathrm{HS}$ in genotypes $B$ and $\mathrm{C} \mathrm{HBV} .{ }^{9}$ Moreover, a recent study demonstrated that HS diagnosed with sonography showed no significant correlation with virological response or HBeAg seroconversion in CHB patients treated with entecavir. ${ }^{10}$ In contrast, pooled data from seven studies showed a strong protective influence of HS on HBV viral load." The unfavorable influence of HS on the clinical outcomes of patients with HBV infection has been also reported. Recent studies strongly suggested that severe HS is associated with severe fibrosis in HBV infection ${ }^{12,13}$ and that HS lowered the efficacy of AVT. ${ }^{10}$ Furthermore, a previous study showed that the presence of metabolic syndrome, which is closely correlated with $\mathrm{HS}$, was an independent risk factor linked with an increased risk of liver cirrhosis and HCC in CHB patients. ${ }^{6,14}$

Liver biopsy is considered the gold standard for evaluating $\mathrm{HS}^{15}$ However, this process cannot be sufficiently used repetitively and its value as a screening method is limited by its drawbacks ranging from invasiveness to sampling errors. ${ }^{16}$ As a noninvasive tool for HS diagnosis, ultrasonography is commonly used; however, it has limitations such as observer variability and low sensitivity of mild HS. ${ }^{17}$ Recently, transient elastography (TE), equipped with a controlled attenuation parameter (CAP), is a proven effective method for determining the degree of HS and fibrosis simultaneously. The accuracy of CAP in detecting HS has been considered acceptable. $^{18}$

Thus, because it is not yet confirmed whether HS can influence the outcome of AVT, we aimed to evaluate the influence of $\mathrm{HS}$, assessed using CAP, on the treatment outcomes in CHB patients initiated on AVT using entecavir and tenofovir.

\section{MATERIALS AND METHODS}

Between 2007 and 2016, treatment-naïve CHB patients with available TE results who were initiated on AVT with entecavir or tenofovir, from the database of Severance Hospital, Yonsei University College of Medicine, Seoul, South Korea, were considered eligible for this retrospective study. CHB was defined as the persistent presence of serum HBV surface antigen for $>6$ months. The exclusion criteria were as follows: liver stiffness (LS) measurement failure; unreliable LS value; $>1$ month interval between AVT initiation and TE assessment; HCC at enrollment or history of HCC; hepatic decompensation or liver transplantation at enrollment, or history of either condition; Child-Pugh class B or C; alanine aminotransferase (ALT) $>5 \times$ the upper normal limit; co-infection with human immunodeficiency virus or hepatitis $\mathrm{C}$ virus; alcohol ingestion in excess of $40 \mathrm{~g} /$ day for $>5$ years; right-sided heart failure; ascites or pregnancy; any other serious medical comorbidities; or follow-up duration $<12$ months.

The study methodology conforms to the ethical guidelines of the Declaration of Helsinki and was approved by the institutional review board of Severance Hospital. Informed consent was waived owing to the retrospective nature of the study.

\section{Enrollment and follow-up}

Patients initiated on AVT with either entecavir or tenofovir were selected according to our inclusion and exclusion criteria. AVT was administered according to the treatment guidelines of the Korean Association for the Study of the Liver ${ }^{19}$ and the reimbursement guidelines of the National Health Insurance Service of the Republic of Korea. After AVT initiation, each patient was regularly followed up every 3 or 6 months with ultrasound examination and laboratory tests. These included tests for the levels of alpha-fetoprotein and virological markers such as HBV DNA and HBeAg and anti-HBe antibody. In case of virological breakthrough (defined as $>1 \log \mathrm{IU} / \mathrm{mL}$ increase in serum HBV DNA level from nadir on 2 consecutive tests) or genotypic mutation, rescue therapy was applied. $^{20}$ 


\section{Fibrosis and steatosis assessment with TE}

TE was performed on the right lobe of the liver through the intercostal spaces, with the patient lying down in the dorsal decubitus position with the right arm in maximal abduction. Experienced nurses blinded to clinical information performed TE (FibroScan ${ }^{\circledR}$; EchoSens, Paris, France). The results were expressed in kilopascals $(\mathrm{kPa})$ for fibrosis and decibels per meter $(\mathrm{dB} / \mathrm{m})$ for steatosis. Interquartile range (IQR) was used as an index of intrinsic variability of TE readings, corresponding to the central interval containing $50 \%$ of valid measurements between the 25th and 75th percentiles. Only examinations with at least 10 validated measurements and a success rate of at least $60 \%$ and an IQR to median ratio $<30 \%$ for LS were accepted as reliable. ${ }^{15,19}$ The cutoff LS value for significant fibrosis was $13 \mathrm{kPa}{ }^{21}$ The cutoff CAP value to diagnose the presence of $\mathrm{HS}$ was $\geq 238 \mathrm{~dB} / \mathrm{m}^{22}$

\section{End points and definitions}

Complete virological response (CVR), HBeAg loss among HBeAg-positive patients, and HCC development were monitored. CVR was defined as undetectable HBV DNA level $<12$ IU/L. HBeAg loss was defined as HBeAg negativity among HBeAg-positive patients at enrollment. HCC diagnosis was based on the guidelines of the American Association for the Study of Liver Diseases. ${ }^{23}$ Liver cirrhosis was diagnosed on the basis of ultrasonographic findings suggestive of cirrhosis, including a blunted, nodular liver edge with splenomegaly $(>12 \mathrm{~cm})^{24}$

\section{Statistical analysis}

Continuous variables were expressed as median with IQR, as appropriate. When comparing baseline characteristics between two groups, a chi-square test (or Fisher's exact test) and Student's $t$-test (or Mann-Whitney U-test) were used. Actuarial rates of clinical outcomes were calculated using the Kaplan-Meier method and compared with the log-rank test. Independent risk factors for each clinical outcome were estimated using multivariate Cox proportional hazard regression analysis. Multivariate analysis was performed using variables with a $P$-value of $<0.2$ in univariate analysis. Statistical analyses were performed using SPSS software 24.0 (IBM Corp., Armonk, NY, USA), and statistical significance was considered for comparisons with a 2-tailed $P$-value of $<0.05$.

\section{RESULTS}

\section{Baseline characteristics}

Among 1,658 treatment-naïve patients with CHB who were initiated on AVT with entecavir or tenofovir between 2007 and 2016, 476 patients with available TE results were evaluated. Twenty-one patients were excluded owing to LS measurement failure or unreliable LS value. Of 455 patients with reliable LS value, 121 were excluded according to our exclusion criteria. Finally, 334 patients were selected for this retrospective study (Supplementary Fig. 1).

The baseline characteristics of the whole study population are described in Table 1. The median age of the study population (210 men and 124 women) was 51.0 years. HBeAg positivity was found in $172(51.5 \%)$ patients, and the median HBV DNA level was 5.84 $\log$ IU/mL. The median CAP value was 229.0 (IQR 202.8-255.0) $\mathrm{dB} / \mathrm{m}$. Most patients $(n=292,87.4 \%$ ) received tenofovir.

\section{Comparison between patients with and without HS}

Of the study population, 146 (43.7\%) patients showed HS (Table 1). When patients with and without HS were compared, patients with HS had significantly higher body mass index (BMI), serum albumin level, platelet count, total cholesterol level, and CAP values than those without HS (all $P<0.05$ ) However, $L S$ values and the proportion of patients with liver cirrhosis ( $45.9 \%$ vs. $51.6 \%$ ) were not significantly different between patients with and without HS (all $P>0.05$ ) (Table 1).

\section{Treatment outcomes of AVT}

The median follow-up period was 38.6 (IQR 28.1-47.6) months (median 38.4 [IQR 28.0-46.7] in patients with HS vs. 38.6 [IQR 28.1-48.4] months in patients without $H S, P=0.757$ ).

The AVT outcomes are listed in Supplementary Table 1. During the follow-up period, $303(90.7 \%)$ and $25(7.5 \%)$ patients achieved CVR and developed HCC at 5 years, respectively. Among 172 HBeAg-positive patients, 37 (21.5\%) experienced HBeAg loss. the proportion of patients with HBeAg loss among HBeAgpositive patients at 5 years was significantly higher in those without HS (28.3\% vs. $13.8 \%$; $P=0.022$, log-rank test) (Supplementary Table 1, Fig. 1).

Among patients treated with entecavir $(n=42,12.6 \%)$, the probability of HBeAg loss among HBeAg-positive patients was 
Table 1. Baseline characteristics of the study populations

\begin{tabular}{|c|c|c|c|c|}
\hline Variables & All patients $(n=334)$ & $\begin{array}{l}\text { Patients with HS } \\
(n=146,43.7 \%)\end{array}$ & $\begin{array}{l}\text { Patients without HS } \\
(n=188,56.3 \%)\end{array}$ & $P$-value \\
\hline \multicolumn{5}{|l|}{ Demographic data } \\
\hline Age (years) & $51.0(42.8-57.0)$ & $51.0(42.0-56.3)$ & $51.0(43.3-57.0)$ & 0.205 \\
\hline Male gender & $210(62.9)$ & $91(62.3)$ & $119(63.3)$ & 0.856 \\
\hline Body mass index $\left(\mathrm{kg} / \mathrm{m}^{2}\right)$ & $23.5(21.6-25.5)$ & $24.7(22.3-26.7)$ & $22.5(20.4-24.4)$ & $<0.001$ \\
\hline Diabetes & $26(7.8)$ & $13(8.9)$ & $13(6.9)$ & 0.501 \\
\hline \multicolumn{5}{|l|}{ Laboratory data } \\
\hline Total bilirubin (mg/dL) & $0.9(0.7-1.1)$ & $0.8(0.7-1.1)$ & $0.9(0.7-1.2)$ & 0.169 \\
\hline Serum albumin (g/dL) & $4.2(3.9-4.4)$ & $4.2(4.0-4.4)$ & $4.2(3.9-4.4)$ & 0.015 \\
\hline Alanine aminotransferase (IU/L) & $56(35-93)$ & $56(38-94)$ & $56(34-90)$ & 0.429 \\
\hline Platelet count $\left(10^{9} / \mathrm{L}\right)$ & $149(111-195)$ & $167(116-215)$ & $144(103-188)$ & 0.003 \\
\hline Prothrombin time (INR) & $1.02(0.96-1.09)$ & $1.01(0.97-1.09)$ & $1.03(0.95-1.10)$ & 0.519 \\
\hline Total cholesterol (mg/dL) & $173(151-196)$ & $178(151-204)$ & $170(151-190)$ & 0.012 \\
\hline Low-density lipoprotein (mg/dL) & $109(90-127)$ & $113(93-129)$ & $101(80-126)$ & 0.293 \\
\hline Fasting glucose (mg/dL) & $95.0(88.3-104.0)$ & $96.0(89.3-107.0)$ & $94.0(87.3-102)$ & 0.040 \\
\hline Alpha-fetoprotein (ng/mL) & $4.7(2.7-9.6)$ & $4.6(2.9-9.4)$ & $5.0(2.5-10.2)$ & 0.100 \\
\hline HBeAg positivity & $172(51.5)$ & $80(54.8)$ & $92(48.9)$ & 0.387 \\
\hline HBV DNA (log IU/mL) & $5.84(4.48-7.13)$ & $6.10(4.01-7.21)$ & $5.7(4.6-7.0)$ & 0.998 \\
\hline Liver cirrhosis & $164(49.1)$ & $67(45.9)$ & $97(51.6)$ & 0.301 \\
\hline \multicolumn{5}{|l|}{ Transient elastography } \\
\hline Liver stiffness (kPa) & $11.1(7.1-16.7)$ & $11.6(7.1-17.0)$ & $10.3(7.1-16.5)$ & 0.391 \\
\hline Liver stiffness $>13 \mathrm{kPa}$ & $132(39.5)$ & $64(43.8)$ & $68(36.2)$ & 0.155 \\
\hline $\mathrm{CAP}(\mathrm{dB} / \mathrm{m})$ & $229.0(202.8-255.0)$ & $261(248-284)$ & 207 (189-223) & $<0.001$ \\
\hline Entecavir/tenofovir & $42(12.6) / 292(87.4)$ & $15(10.3) / 131(89.7)$ & $27(14.4) / 161(85.6)$ & 0.264 \\
\hline
\end{tabular}

Variables are expressed as median (interquartile range) or $\mathrm{n}(\%)$.

HS, hepatic steatosis; INR, international normalized ratio; HBeAg, hepatitis B e antigen; HBV, hepatitis B virus; kPa, kilopascal; CAP, controlled attenuation parameter; $\mathrm{dB} / \mathrm{m}$, decibels per meter.
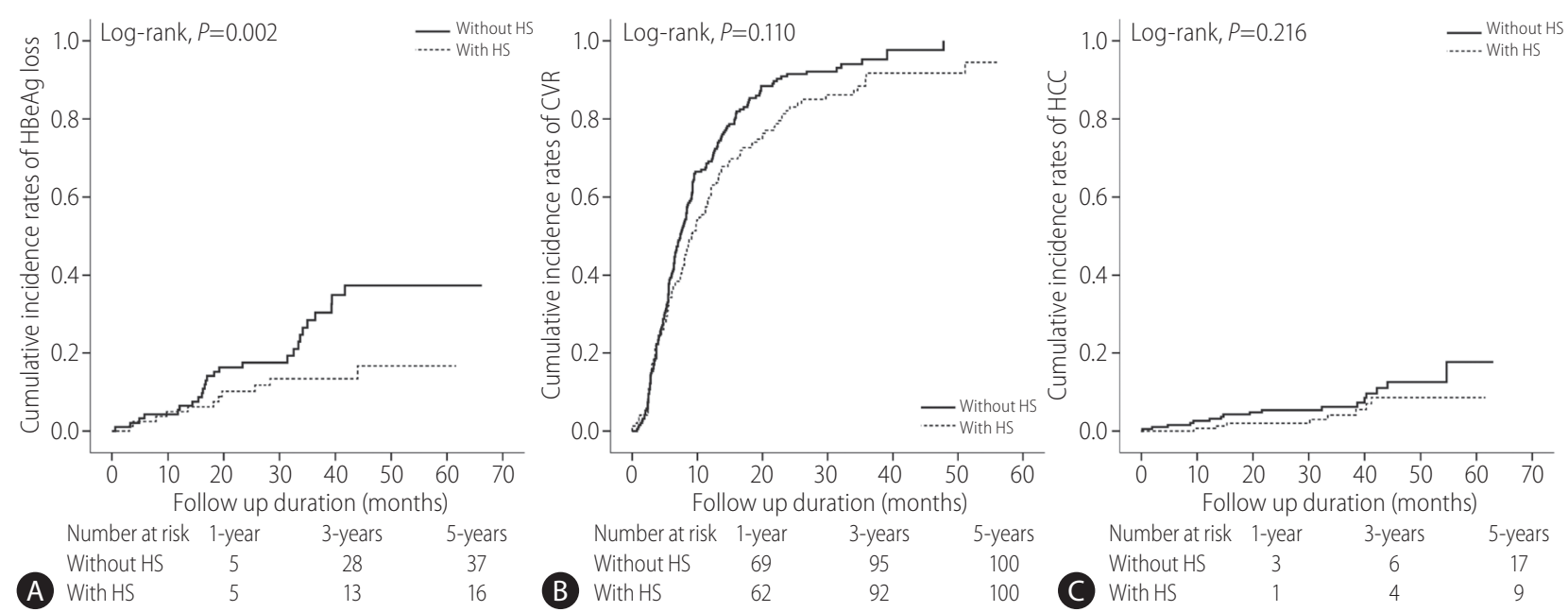

$\begin{array}{lccc}\text { Number at risk } & 1 \text {-year } & 3 \text {-years } & 5 \text {-years } \\ \text { Without HS } & 69 & 95 & 100 \\ \text { With HS } & 62 & 92 & 100\end{array}$

$\begin{array}{lccc}\text { Number at risk } & \text { 1-year } & \text { 3-years } & \text { 5-years } \\ \text { Without HS } & 3 & 6 & 17 \\ \text { C. With HS } & 1 & 4 & 9\end{array}$

Figure 1. Cumulative incidence rates of HBeAg loss (A) ( $n=172)$, CVR (B), and HCC (C) ( $n=334)$ in patients with and without HS (Kaplan-Meier plot). The cumulative incidence rates of $\mathrm{HBeAg}$ loss were calculated among HBeAg-positive patients. HBeAg, hepatitis B e antigen; CVR, complete virological response; HCC, hepatocellular carcinoma; HS, hepatic steatosis. 
not statistically significant between the groups with and without HS ( $P=0.139$, log-rank test), whereas among patients treated with tenofovir ( $n=292,87.4 \%$ ), the probability of HBeAg loss was significantly higher in the group without HS ( $P=0.046$, log-rank test) (Supplementary Fig. 2).

The follow-up period was statistically similar between HBeAgpositive and HBeAg-negative patients (median 38.7 [IQR 28.348.0] vs. 38.6 [IQR 27.9-47.4] months, $P=0.461$ ). In contrast, the follow-up duration of patients treated with entecavir and tenofovir was significantly different (median 47.0 [IQR 39.1-59.0] vs. 36.7 [27.3-46.7] months, $P<0.001)$. When the study population was stratified according to HBeAg status, the proportion of patients with CVR achievement was significantly higher at 5 years among $\mathrm{HBeAg}$-negative patients than in $\mathrm{HBeAg}$-positive patients (96.3\% vs. 85.5\%; $P<0.001$, log-rank test) (Supplementary Table 2, Supplementary Fig. 3).

\section{Comparisons between patients with and without HBeAg loss, CVR achievement, and HCC development}

Because the rate of $\mathrm{HBeAg}$ loss was significantly different according to the presence of $\mathrm{HS}$, we selected $172 \mathrm{HBeAg}$-positive patients and compared the baseline characteristics between those with and without HBeAg loss (Table 2). Patients with HBeAg loss showed significantly lower CAP value (median 214.0 vs. 239.0 $\mathrm{dB} / \mathrm{m}, P=0.013)$, whereas the proportion of CVR achievement was significantly higher in patients with HBeAg loss (97.3 vs. 82.2\%, $P=0.021$ ) (Table 2).

When the study population was stratified according to CVR achievement (Table 3) and HCC development (Supplementary Table 3), BMI (median 23.3 vs. $24.9 \mathrm{~kg} / \mathrm{m}^{2}$ ), ALT level (median 54 vs. $66 \mathrm{IU} / \mathrm{L}$ ), platelet count (median $148 \times 10^{9} / \mathrm{L}$ vs. $\left.182 \times 10^{9} / \mathrm{L}\right), \mathrm{HBeAg}$

Table 2. Comparison between patients with and without HBeAg loss among HBeAg-positive patients ( $n=172)$

\begin{tabular}{|c|c|c|c|}
\hline Variables & HBeAg loss ( $n=37,21.5 \%)$ & No HBeAg loss ( $n=135,78.5 \%)$ & $P$-value \\
\hline \multicolumn{4}{|l|}{ Demographic data } \\
\hline Age (years) & $45.0(36.5-54.5)$ & $48.0(40.0-56.0)$ & 0.401 \\
\hline Male gender & $20(54.1)$ & $81(60.0)$ & 0.515 \\
\hline Body mass index $\left(\mathrm{kg} / \mathrm{m}^{2}\right)$ & $23.6(22.1-25.2)$ & $23.4(20.8-25.6)$ & 0.725 \\
\hline Diabetes & $5(13.5)$ & $10(7.4)$ & 0.320 \\
\hline \multicolumn{4}{|l|}{ Laboratory data } \\
\hline Total bilirubin (mg/dL) & $0.9(0.6-1.1)$ & $0.8(0.6-1.1)$ & 0.344 \\
\hline Serum albumin (g/dL) & $4.1(3.8-4.3)$ & $4.1(3.9-4.3)$ & 0.252 \\
\hline Alanine aminotransferase (IU/L) & $59(44-119)$ & $56(37-88)$ & 0.088 \\
\hline Platelet count $\left(10^{9} / \mathrm{L}\right)$ & 149 (105-199) & $150(112-211)$ & 0.542 \\
\hline Prothrombin time (INR) & $1.06(1.00-1.13)$ & $1.02(0.95-1.09)$ & 0.812 \\
\hline Total cholesterol (mg/dL) & $170.0(155.0-182.5)$ & $174.0(154.0-196.5)$ & 0.356 \\
\hline Low-density lipoprotein (mg/dL) & $125.0(91.0-161.3)$ & $108.5(87.3-130.3)$ & 0.319 \\
\hline Alpha-fetoprotein (ng/mL) & $5.2(2.8-10.4)$ & $6.1(3.6-13.0)$ & 0.479 \\
\hline HBV DNA (log IU/mL) & $6.31(4.95-7.83)$ & $7.0(5.8-8.2)$ & 0.130 \\
\hline Liver cirrhosis & $5(13.5)$ & $5(3.7)$ & 0.248 \\
\hline \multicolumn{4}{|l|}{ Transient elastography } \\
\hline Liver stiffness (kPa) & $11.8(7.7-16.4)$ & $11.9(7.1-18.8)$ & 0.535 \\
\hline Liver stiffness $>13 \mathrm{kPa}$ & $15(40.5)$ & $63(46.7)$ & 0.507 \\
\hline $\mathrm{CAP}(\mathrm{dB} / \mathrm{m})$ & $214.0(195.0-251.0)$ & $239.0(204.0-260.0)$ & 0.013 \\
\hline Complete virological response & $36(97.3)$ & $111(82.2)$ & 0.021 \\
\hline Tenofovir (vs. entecavir) & $33(89.2) / 4(10.8)$ & $119(88.1) / 16(11.9)$ & 0.562 \\
\hline
\end{tabular}

Variables are expressed as median (interquartile range) or $\mathrm{n}(\%)$.

HBeAg, hepatitis B e antigen; INR, international normalized ratio; HBV, hepatitis B virus; kPa, kilopascal; CAP, controlled attenuated parameter; dB/m, decibels per meter. 
positivity (48.5 vs. 80.6\%), HBV DNA level (median 5.66 vs. 8.23 $\log \mathrm{IU} / \mathrm{mL}$ ), CAP value (median 228 vs. $244 \mathrm{~dB} / \mathrm{m}$ ) were significantly lower in patients with CVR achievement than in those without (all $P<0.05$ ), whereas the proportion of liver cirrhosis was significantly higher in patients with CVR achievement than in those without (51.2 vs. $27.3 \%, P=0.019$ ). Additionally, patients who developed HCC were significantly older (median age 57.0 vs. 50.0 years) and had a significantly higher proportion of liver cirrhosis (92.0\% vs. $45.6 \%$ ) than those who did not (all $P<0.001$ ).

\section{Independent predictors of treatment outcomes}

First, univariate and subsequent multivariate analyses were performed to identify independent predictors of HBeAg loss among HBeAg-positive patients (Table 4). When CAP values were adjust- ed as continuous variables, higher ALT level (hazard ratio $[H R]=1.017,95 \%$ confidence interval $[\mathrm{Cl}] 1.008-1.027 ; P<0.001)$, lower HBV DNA level $(H R=0.814,95 \% \mathrm{Cl} 0.687-0.964$; $P=0.017)$, and lower CAP values ( $\mathrm{HR}=0.991,95 \% \mathrm{Cl} 0.983-$ 0.999; $P=0.026$ ) were independently associated with a higher probability of HBeAg loss among HBeAg-positive patients. Furthermore, CAP value was selected as an independent predictor of CVR achievement (Table 5, Supplementary Table 4).

When the cutoff value of $238 \mathrm{~dB} / \mathrm{m}$ was used to define HS (S1-3 $[n=146,43.7 \%]$ vs. SO $[n=188,56.3 \%])^{22}$ the presence of HS tended to reduce the probability of HBeAg loss ( $H R=0.494,95 \%$ $\mathrm{Cl}$ 0.233-1.047, $P=0.066)$; however, both CVR achievement and HCC development were not significantly associated with HS after adjustment (all $P>0.1$ ). In addition, when HS was further divided as $50-1$ ( $n=259,77.5 \%)$ vs. $S 2-3(n=75,22.5 \%)$ using the cutoff

Table 3. Comparison between patients with and without CVR

\begin{tabular}{|c|c|c|c|}
\hline Variables & $\begin{array}{l}\text { Patients with CVR } \\
(n=303,90.7 \%)\end{array}$ & $\begin{array}{l}\text { Patients without CVR } \\
\qquad(n=33,9.3 \%)\end{array}$ & $P$-value \\
\hline \multicolumn{4}{|l|}{ Demographic data } \\
\hline Age (years) & $51.0(44.0-57.0)$ & $41.0(33.0-57.0)$ & 0.069 \\
\hline Male gender & $191(63.0)$ & $19(61.3)$ & 0.848 \\
\hline Body mass index $\left(\mathrm{kg} / \mathrm{m}^{2}\right)$ & $23.3(21.5-25.4)$ & $24.9(22.7-26.6)$ & 0.033 \\
\hline Diabetes & $23(7.6)$ & $3(9.7)$ & 0.721 \\
\hline \multicolumn{4}{|l|}{ Laboratory data } \\
\hline Total bilirubin (mg/dL) & $0.9(0.7-1.1)$ & $0.8(0.6-1.2)$ & 0.519 \\
\hline Serum albumin (g/dL) & $4.2(3.9-4.4)$ & $4.1(4.0-4.3)$ & 0.874 \\
\hline Alanine aminotransferase (IU/L) & $54(34-91)$ & $66(48-119)$ & 0.040 \\
\hline Platelet count $\left(10^{9} / \mathrm{L}\right)$ & $148(110-192)$ & $182(118-236)$ & 0.044 \\
\hline Prothrombin time (INR) & $1.02(0.96-1.09)$ & $1.01(0.94-1.16)$ & 0.770 \\
\hline Total cholesterol (mg/dL) & $172(151-196)$ & $174(150-193)$ & 0.923 \\
\hline Low-density lipoprotein (mg/dL) & $112(92-178)$ & $102(79.8-135.3)$ & 0.743 \\
\hline Alpha-fetoprotein (ng/mL) & $4.73(2.68-9.48)$ & $4.1(2.6-12.5)$ & 0.924 \\
\hline HBeAg positivity & $147(48.5)$ & 25 (80.6) & 0.001 \\
\hline HBV DNA (log IU/mL) & $5.66(4.25-6.87)$ & $8.23(6.74-8.23)$ & $<0.001$ \\
\hline Liver cirrhosis & $155(51.2)$ & $9(27.3)$ & 0.019 \\
\hline \multicolumn{4}{|l|}{ Transient elastography } \\
\hline Liver stiffness (kPa) & $10.9(7.1-16.8)$ & $11.6(6.3-16.6)$ & 0.657 \\
\hline Liver stiffness $>13 \mathrm{kPa}$ & $121(39.9)$ & $11(35.5)$ & 0.629 \\
\hline $\mathrm{CAP}(\mathrm{dB} / \mathrm{m})$ & $228(202-253)$ & $244(214-279)$ & 0.044 \\
\hline Tenofovir (vs. entecavir) & $264(87.1) / 39(12.9)$ & $28(90.3) / 3(9.7)$ & 0.780 \\
\hline
\end{tabular}

Variables are expressed as median (interquartile range) or $\mathrm{n}(\%)$.

CVR, complete virological response; INR, international normalized ratio; HBeAg, hepatitis B e antigen; HBV, hepatitis B virus; $\mathrm{KPa}$, kilopascal; CAP, controlled attenuated parameter; $\mathrm{dB} / \mathrm{m}$, decibels per meter. 
value of $269 \mathrm{~dB} / \mathrm{m}_{1}^{22} \mathrm{S2}-3$ was not associated with the probability of HBeAg loss and HCC development (all $P>0.1$ ). S2-3 was, however, independently associated with a reduced probability of CVR achievement (HR=0.676, 95\% Cl 0.500-0.914, $P=0.011$ ).

\section{DISCUSSION}

In this study, around half of the study population with CHB had concomitant HS (146 of 334, 43.7\%) at the time of initiating AVT. During the study period, 303 (90.7\%) and 25 (7.5\%) patients achieved CVR and developed HCC, respectively. Moreover, among 172 HBeAg-positive patients, 37 (21.5\%) experienced HBeAg loss. Multivariate analysis indicated that lower CAP value was independently associated with a higher probability of $\mathrm{HBeAg}$ loss among $\mathrm{HBeAg}$-positive patients $(\mathrm{HR}=0.989)$ and with $C V R$ achievement in the entire study population ( $H R=0.996, P=0.004)$, whereas the probability of HCC development was not significantly associated with CAP value $(P=0.380)$. Finally, the cumulative incidence of HBeAg loss among HBeAg-positive patients was significantly higher in patients without HS than in those with HS ( $P=0.022$, log-rank test).

In accordance with the rising prevalence of fatty liver worldwide, the prevalence of concomitant HS in chronic viral hepatitis and its influence on the natural course or treatment outcomes have recently gained medical interest. ${ }^{6}$ Furthermore, recent potent antiviral agents such as entecavir and tenofovir have been reported to suppress viral replication and the progression of liver cirrhosis, in of patients treated with AVT. ${ }^{25}$ Thus, our study naturally focused on the remaining factors of concomitant $\mathrm{HS}$ that could not be controlled using antiviral agents. According to previous studies in CHB patients, the prevalence of concomitant HS has ranged

Table 4. Independent predictors of HBeAg loss among HBeAg-positive patients

\begin{tabular}{|c|c|c|c|}
\hline \multirow{2}{*}{ Variables } & \multirow{2}{*}{$\begin{array}{c}\text { Univariate } \\
P \text {-value }\end{array}$} & \multicolumn{2}{|c|}{ Multivariate (adjusting CAP value) } \\
\hline & & $\mathrm{HR}(95 \% \mathrm{Cl})$ & $P$-value \\
\hline \multicolumn{4}{|l|}{ Demographic data } \\
\hline Age (years) & 0.498 & - & - \\
\hline Male gender & 0.524 & - & - \\
\hline Body mass index $\left(\mathrm{kg} / \mathrm{m}^{2}\right)$ & 0.629 & - & - \\
\hline Diabetes & 0.221 & - & - \\
\hline \multicolumn{4}{|l|}{ Laboratory data } \\
\hline Total bilirubin (mg/dL) & 0.219 & - & - \\
\hline Serum albumin (g/dL) & 0.121 & $0.669(0.346-1.292)$ & 0.231 \\
\hline Alanine aminotransferase (IU/L) & 0.063 & $1.017(1.008-1.027)$ & $<0.001$ \\
\hline Platelet count $\left(10^{9} / \mathrm{L}\right)$ & 0.342 & - & - \\
\hline Prothrombin time (INR) & 0.610 & - & - \\
\hline Total cholesterol (mg/dL) & 0.281 & - & - \\
\hline Low-density lipoprotein (mg/dL) & 0.309 & - & - \\
\hline Alpha-fetoprotein (ng/mL) & 0.571 & - & - \\
\hline HBV DNA (log IU/mL) & 0.159 & $0.814(0.687-0.964)$ & 0.017 \\
\hline Liver cirrhosis & 0.152 & $2.064(0.955-4.459)$ & 0.065 \\
\hline \multicolumn{4}{|l|}{ Transient elastography } \\
\hline Liver stiffness (kPa) & 0.654 & - & - \\
\hline Liver stiffness $>13 \mathrm{kPa}$ & 0.694 & - & - \\
\hline $\mathrm{CAP}(\mathrm{dB} / \mathrm{m})$ & 0.021 & $0.991(0.983-0.999)$ & 0.026 \\
\hline Tenofovir (vs. entecavir) & 0.598 & - & - \\
\hline
\end{tabular}

Variables are expressed as median (interquartile range) or $n(\%)$.

HBeAg, hepatitis B e antigen; CAP, controlled attenuated parameter; HR, hazard ratio; Cl, confidence interval; INR, international normalized ratio; HBV, hepatitis $\mathrm{B}$ virus; $\mathrm{KPa}$, kilopascal; $\mathrm{dB} / \mathrm{m}$, decibels per meter. 
from $15 \%$ to $48.8 \%{ }^{6,26}$ The prevalence of HS affects $20-46 \%$ of the general Western and Asian population, 12,27 which is not so different from that estimated in CHB patients with HS. In our cohort, $43.7 \%$ of the CHB population had coexisting HS, which is similar to the previous prevalence.

Studies on the interaction between HS and HBV have provided controversial results. Pooled data of seven studies found an inverse association between HBV DNA levels and fatty liver in HBV patients, ${ }^{11}$ whereas Wong et al. reported a negative association between $\mathrm{CHB}$ and nonalcoholic fatty liver disease prevalence. ${ }^{28}$ Our study found a significant influence of HS on the long-term treatment outcomes of AVT in CHB patients; higher CAP values were associated with a lower probability of HBeAg loss among HBeAg-positive patients. Our results are supported by a recent meta-analysis showing that the efficacy of AVT declined in CHB patients with $\mathrm{HS}^{10}$ This study suggested that virological and bio- chemical responses were significantly different between subgroups with and without HS diagnosed using sonography. ${ }^{19}$ This negative effect of HS on HBeAg loss in HBeAg-positive patients might be explained by the decreased bioavailability of AVT due to fatty liver load and its fatty acid deposits leading to a significant loss in contact area between hepatocytes and antiviral agents. ${ }^{29}$ In contrast, other studies have indicated that HS would not be significantly associated with virological response or HBeAg seroconversion. ${ }^{26}$ Differences in antiviral agents, range of $\mathrm{BMI}$, or diagnostic tools for diagnosing HS (TE in our study vs. ultrasonography in previous studies) might explain this discrepant result. Indeed, TE has been considered a more reliable tool for the diagnosis and grading of HS in chronic viral hepatitis. ${ }^{17}$

Furthermore, CAP value was selected as an independent negative predictor of CVR achievement in the entire study population. Cell histological changes in fatty liver, such as the nucleus being

Table 5. Independent predictors of complete virological response

\begin{tabular}{|c|c|c|c|}
\hline \multirow{2}{*}{ Variables } & \multirow{2}{*}{$\begin{array}{c}\text { Univariate } \\
P \text {-value }\end{array}$} & \multicolumn{2}{|c|}{ Multivariate (adjusting CAP value) } \\
\hline & & $\mathrm{HR}(95 \% \mathrm{Cl})$ & $P$-value \\
\hline \multicolumn{4}{|l|}{ Demographic data } \\
\hline Age (years) & 0.001 & $0.999(0.987-1.011)$ & 0.838 \\
\hline Male gender & 0.846 & - & - \\
\hline Body mass index $\left(\mathrm{kg} / \mathrm{m}^{2}\right)$ & 0.328 & - & - \\
\hline Diabetes & 0.818 & - & - \\
\hline \multicolumn{4}{|l|}{ Laboratory data } \\
\hline Total bilirubin (mg/dL) & 0.250 & - & - \\
\hline Serum albumin (g/dL) & 0.156 & $1.366(1.014-1.840)$ & 0.040 \\
\hline Alanine aminotransferase (IU/L) & 0.003 & $0.999(0.995-1.002)$ & 0.458 \\
\hline Platelet count $\left(10^{9} / \mathrm{L}\right)$ & $<0.001$ & $0.998(0.996-1.000)$ & 0.075 \\
\hline Prothrombin time (INR) & 0.722 & - & - \\
\hline Total cholesterol (mg/dL) & 0.223 & - & - \\
\hline Low-density lipoprotein (mg/dL) & 0.884 & - & - \\
\hline Alpha-fetoprotein (ng/mL) & 0.311 & - & - \\
\hline HBeAg positivity & $<0.001$ & $0.601(0.451-0.800)$ & $<0.001$ \\
\hline HBV DNA (log IU/mL) & $<0.001$ & $0.794(0.738-0.854)$ & $<0.001$ \\
\hline Liver cirrhosis & $<0.001$ & $0.957(0.724-1.265)$ & 0.759 \\
\hline \multicolumn{4}{|l|}{ Transient elastography } \\
\hline Liver stiffness (kPa) & 0.782 & - & - \\
\hline Liver stiffness $>13 \mathrm{kPa}$ & 0.605 & - & - \\
\hline $\mathrm{CAP}(\mathrm{dB} / \mathrm{m})$ & 0.018 & 0.996 (0.993-0.999) & 0.004 \\
\hline Tenofovir (vs. entecavir) & 0.808 & - & - \\
\hline
\end{tabular}

CAP, controlled attenuated parameter; HR, hazard ratio; $\mathrm{Cl}$, confidence interval; INR, international normalized ratio; HBeAg, hepatitis B e antigen; HBV hepatitis $\mathrm{B}$ virus; $\mathrm{KPa}$, kilopascal; $\mathrm{dB} / \mathrm{m}$, decibels per meter. 
pushed to the periphery, could prevent the interaction between the AVT agent and viral nucleic acids and explain the negative correlation between CAP value and the probability of CVR achievement. ${ }^{30}$ After adjustment, HBeAg positivity and HBV DNA levels were both significantly associated with a lower probability of CVR achievement, which has been a well-known disadvantage. $^{4}$

Although the clear relation between HCC in HS patients and HBV is unclear, many factors, including metabolic factors, that are significantly associated with fatty liver, are considered to increase the risk of HCC development in HBV-infected patients. ${ }^{9}$ However, fatty liver and CAP value did not influence the risk of HCC development in our study. The reason for this discrepancy is unclear, but can be explained in part by the proportion of CHB patients with accompanying nonalcoholic steatohepatitis with fibrosis progression. Indeed, although a recent study by Seto et al. showed a significant correlation between the degree of liver steatosis and fibrosis assessed using $\mathrm{TE}_{1}^{12}$ the association was not significant in our study. Although histological information is lacking, the different proportions of CHB patients with concomitant insult from fatty liver-related inflammation and fibrosis may have been a factor." Thus, the exact association between steatotic burden and the risk of HCC development should be further investigated.

Our study has several advantages that strengthen its clinical implications. First, our study is the first of its kind to comprehensively analyze the effects of HS on the clinical outcomes of CHB patients undergoing treatment with potent AVT agents according to recent guidelines. ${ }^{4}$ Thus, our results can be applied to CHB patients in this era of potent and active AVT. Second, in contrast to previous research with subjective ultrasonography assessment of steatotic burden, our study adopted TE with CAP to assess HS, which has been widely used to assess the degree of fatty liver. 12,18 Finally, we focused on the prognostic clinical influence of accompanying HS in CHB patients undergoing AVT beyond the crosssectional analysis. Although the influence of dynamic change in steatotic burden during AVT could not be assessed, our results might provide the rationale to reduce liver steatosis, to increase the probability of $\mathrm{HBeAg}$ seroconversion for HBeAg-positive patients.

Our study also has several limitations. First, although the overall sample size was acceptable, the proportion of end points such as HCC was relatively small. Thus, the lack of association between steatotic burden and the risk of HCC development might stem from statistical bias. Indeed, cirrhosis being selected as one of the independent predictors of HCC development in our study whereas
$L S$ value assessed using TE was not may be attributable to the small number of patients who developed HCC. Additionally, the high proportion of patients with liver cirrhosis (49.1\%) and high ALT levels at the time of starting AVT, in spite of the exclusion of ALT $>5 \times$ the upper limit of normal, might also have influenced our results. Second, our study was limited by the lack of data on detailed histological characteristics related to the risk of end-point achievement. We do not know whether pure steatotic burden was significantly associated with the treatment outcomes or whether a combination of steatotic burden and additional characteristics such as fibrosis are involved. Lastly, the skewed distribution of patients with different steatotic burden might have biased our results. Owing to the retrospective nature of this study, it was inevitable to have more patients with lesser steatotic burden. Likely due to the same reason, the results from binary stratification of HS using several previous cutoff values were similar, but not consistent with those from continuously expressed CAP values. ${ }^{22}$

In conclusion, we demonstrated that CAP value was not correlated with HCC development in patients initiated on AVT with entecavir and tenofovir. However, CAP value was negatively correlated with the risk of HBeAg loss among HBeAg-positive patients and with CVR achievement in the entire study population.

\section{Author's contribution}

S.U. Kim designed this study.

D.S. Kim, M.Y. Jeon, and S.U. Kim, carried out the data analysis and wrote the manuscript.

D.S. Kim, M.Y. Jeon, H.W. Lee, B.K. Kim, J.Y. Park, D.Y. Kim, S.H. Ahn, K.H. Han, and S.U. Kim contributed to inclusion of patients, acquisition and analysis of data.

All authors contributed to the interpretation of results, critical revision of the manuscript and approved the final manuscript.

S.U. Kim is the guarantor.

\section{Financial support}

This study was supported by the Basic Science Research Program through the National Research Foundation of Korea funded by the Ministry of Science, ICT \& Future Planning (2016R1A1A1A05005138). The funders had no role in the study design, data collection and analysis, decision to publish, or preparation of the manuscript.

\section{Illustration assistance}

The authors are grateful to Dong-Su Jang, (Medical Illustrator, Medical Research Support Section, Yonsei University College of Medicine, Seoul, Republic of Korea) for his help with the figures. 


\section{Conflicts of Interest}

None to declare for all authors.

\section{SUPPLEMENTARY MATERIALS}

Supplementary materials are available at Clinical and Molecular Hepatology website (http://www.e-cmh.org).

\section{REFERENCES}

1. World Health Organization. Hepatitis B vaccines: WHO position paper, July 2017 - Recommendations. Vaccine 2019;37:223-225.

2. Ikeda K, Saitoh S, Suzuki Y, Kobayashi M, Tsubota A, Koida I, et al. Disease progression and hepatocellular carcinogenesis in patients with chronic viral hepatitis: a prospective observation of 2215 patients. J Hepatol 1998;28:930-938.

3. Sookoian S, Pirola CJ. Genetic predisposition in nonalcoholic fatty liver disease. Clin Mol Hepatol 2017;23:1-12.

4. European Association for the Study of the Liver. EASL 2017 Clinical Practice Guidelines on the management of hepatitis B virus infection. J Hepatol 2017;67:370-398.

5. Snow-Lampart A, Chappell B, Curtis M, Zhu Y, Myrick F, Schawalder $J$, et al. No resistance to tenofovir disoproxil fumarate detected after up to 144 weeks of therapy in patients monoinfected with chronic hepatitis B virus. Hepatology 2011;53:763-773.

6. Fan JG, Kim SU, Wong VW. New trends on obesity and NAFLD in Asia. J Hepatol 2017;67:862-873.

7. Wang CC, Hsu CS, Liu CJ, Kao JH, Chen DS. Association of chronic hepatitis B virus infection with insulin resistance and hepatic steatosis. J Gastroenterol Hepatol 2008;23:779-782.

8. Shi JP, Fan JG, Wu R, Gao XQ, Zhang L, Wang H, et al. Prevalence and risk factors of hepatic steatosis and its impact on liver injury in Chinese patients with chronic hepatitis B infection. J Gastroenterol Hepatol 2008;23:1419-1425.

9. Wong VW, Wong GL, Yu J, Choi PC, Chan AW, Chan HY, et al. Interaction of adipokines and hepatitis B virus on histological liver injury in the Chinese. Am J Gastroenterol 2010;105:132-138.

10. Zhu Y, Yang Q, Lv F, Yu Y. The effect of hepatosteatosis on response to antiviral treatment in patients with chronic hepatitis B: a metaanalysis. Gastroenterol Res Pract 2017;2017:1096406.

11. Machado MV, Oliveira AG, Cortez-Pinto H. Hepatic steatosis in hepatitis B virus infected patients: meta-analysis of risk factors and comparison with hepatitis C infected patients. J Gastroenterol Hepatol 2011;26:1361-1367.

12. Seto WK, Hui RWH, Mak LY, Fung J, Cheung KS, Liu KSH, et al.
Association between hepatic steatosis, measured by controlled attenuation parameter, and fibrosis burden in chronic hepatitis B. Clin Gastroenterol Hepatol 2018;16:575-583.e2.

13. Wong GL, Wong VW, Choi PC, Chan AW, Chim AM, Yiu KK, et al. Metabolic syndrome increases the risk of liver cirrhosis in chronic hepatitis B. Gut 2009;58:111-117.

14. Chon YE, Kim KJ, Jung KS, Kim SU, Park JY, Kim DY, et al. The relationship between type 2 diabetes mellitus and non-alcoholic fatty liver disease measured by controlled attenuation parameter. Yonsei Med J 2016;57:885-892.

15. Kim BK, Kim HS, Yoo EJ, Oh EJ, Park JY, Kim DY, et al. Risk assessment of clinical outcomes in Asian patients with chronic hepatitis B using enhanced liver fibrosis test. Hepatology 2014;60:1911-1919.

16. Seo YS, Kim MN, Kim SU, Kim SG, Um SH, Han KH, et al. Risk assessment of hepatocellular carcinoma using transient elastography vs. liver biopsy in chronic hepatitis B patients receiving antiviral therapy. Medicine (Baltimore) 2016;95:e2985.

17. Lee DH. Imaging evaluation of non-alcoholic fatty liver disease: focused on quantification. Clin Mol Hepatol 2017;23:290-301.

18. Ahn JM, Paik YH, Min SY, Cho JY, Sohn W, Sinn DH, et al. Relationship between controlled attenuation parameter and hepatic steatosis as assessed by ultrasound in alcoholic or nonalcoholic fatty liver disease. Gut Liver 2016;10:295-302.

19. Ceylan B, Arslan F, Batırel A, Fincancı M, Yardımcı C, Fersan E, et al. Impact of fatty liver on hepatitis $B$ virus replication and virologic response to tenofovir and entecavir. Turk J Gastroenterol 2016;27:42 46.

20. Lok AS, Zoulim F, Locarnini S, Bartholomeusz A, Ghany MG, Pawlotsky JM, et al. Antiviral drug-resistant HBV: standardization of nomenclature and assays and recommendations for management. Hepatology 2007;46:254-265.

21. Kim MN, Kim SU, Kim BK, Park JY, Kim DY, Ahn SH, et al. Increased risk of hepatocellular carcinoma in chronic hepatitis B patients with transient elastography-defined subclinical cirrhosis. Hepatology 2015:61:1851-1859.

22. Kim JK, Lee KS, Choi JR, Chung HJ, Jung DH, Lee KA, et al. Usefulness of the controlled attenuation parameter for detecting liver steatosis in health checkup examinees. Gut Liver 2015;9:405-410.

23. Bruix J, Sherman M; American Association for the Study of Liver Diseases. Management of hepatocellular carcinoma: an update. Hepatology 2011;53:1020-1022.

24. Jung KS, Kim SU, Ahn SH, Park YN, Kim DY, Park JY, et al. Risk assessment of hepatitis B virus-related hepatocellular carcinoma development using liver stiffness measurement (FibroScan). Hepatology 2011;53:885-894.

25. Marcellin P, Gane E, Buti M, Afdhal N, Sievert W, Jacobson IM, et al. Regression of cirrhosis during treatment with tenofovir disoproxil fumarate for chronic hepatitis B: a 5-year open-label follow-up study. 
David Sooik Kim, et al.

Influence of steatosis in patients with chronic hepatitis B

Lancet 2013;381:468-475.

26. Zhu LY, Wang YG, Wei LQ, Zhou J, Dai WJ, Zhang XY. The effects of the insulin resistance index on the virologic response to entecavir in patients with $\mathrm{HBeAg}$-positive chronic hepatitis B and nonalcoholic fatty liver disease. Drug Des Devel Ther 2016;10:2739-2744.

27. Seto WK, Yuen MF. Nonalcoholic fatty liver disease in Asia: emerging perspectives. J Gastroenterol 2017;52:164-174.

28. Wong VW, Wong GL, Chu WC, Chim AM, Ong A, Yeung DK, et al.
Hepatitis B virus infection and fatty liver in the general population. J Hepatol 2012;56:533-540.

29. Lau E, Carvalho D, Freitas P. Gut microbiota: association with NAFLD and metabolic disturbances. Biomed Res Int 2015;2015:979515.

30. Takahashi Y, Fukusato T. Histopathology of nonalcoholic fatty liver disease/nonalcoholic steatohepatitis. World J Gastroenterol 2014;20:15539-15548. 